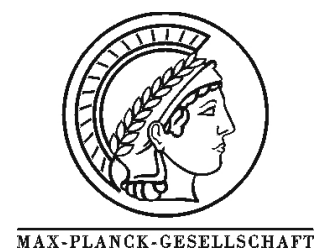

\title{
Transmission Electron Microscopical Studies of the Layered Structure of the Ternary Semiconductor Culn ${ }_{5} \mathrm{Se}_{8}$
}

\author{
A.-T. Tham ${ }^{1}$, D. S. Su ${ }^{2}$, W. Neumann ${ }^{1}$, P. Schubert-Bischoff ${ }^{3}$, C. Beilharz ${ }^{4}$ and K.W. Benz ${ }^{4}$ \\ ${ }^{1}$ Humboldt University of Berlin, Institute of Physics, Chair of Crystallography, Invalidenstr. 110, \\ D-10115 Berlin, Germany \\ ${ }^{2}$ Department of Inorganic Chemistry, Fritz-Haber-Institute of the MPG, Faradayweg 4-6, 14195 Berlin, Germany \\ ${ }^{3}$ Germany Hahn Meitner Institute Berlin GmbH, Glienicker Str. 100, D-14109 Berlin, Germany \\ ${ }^{4}$ University of Freiburg, Institute of Crystallography, Hebelstr. 25, D-79108 Freiburg, Germany
}

Received 01 November 2000; accepted 28 February 2001

\begin{abstract}
The structure of the off-stoichiometric In-rich ternary phase $\mathrm{CuIn}_{5} \mathrm{Se}_{8}$ was studied by means of electron diffraction and high-resolution electron microscopy. The compound shows a layered structure with a 7-layer stacking sequence of closed-packed planes, which contains both cubic and hexagonal stacking of Se atoms. The studied $\mathrm{CuIn}_{5} \mathrm{Se}_{8}$ bulk crystal is known as the $\beta$-phase of this compound.
\end{abstract}

Keywords: TEM, CulnSe, layer

\section{Introduction}

Compounds of the $\mathrm{Cu}-\mathrm{In}-\mathrm{Se}$ system are semiconductor materials with promising applications to solar energy conversion [BLOSS]. Many In-rich phases have been found in this system, one of them is the $\mathrm{CuIn}_{5} \mathrm{Se}_{8}$ phase. The offstoichiometric In-rich $\mathrm{CuIn}_{5} \mathrm{Se}_{8}$ phase is located directly on the $\mathrm{Cu}_{2} \mathrm{Se}-\mathrm{In}_{2} \mathrm{Se}_{3}$ quasibinary section in the ternary phase diagram of the $\mathrm{Cu}$-In-Se system and belongs to the so-called on-tie-line phases. This phase is n-type conducting and electrically compensated, thus a useful partner of p-type $\mathrm{CuInSe}_{2}$ in the fabrication of even more efficient heterojunction solar cells is given [THAM].

The structure of this compound was investigated and reported by several authors (e.g., [SU], [TSENG]). There are known two modifications of this compound: $\alpha-\mathrm{CuIn}_{5} \mathrm{Se}_{8}$ with a tetragonal structure related to the chalcopyrite structure [MANOLIKAS] and $\beta-\mathrm{CuIn}_{5} \mathrm{Se}_{8}$ with a layered structure [FRANGIS].

In the present work the layered structure of $\beta$ $\mathrm{CuIn}_{5} \mathrm{Se}_{8}$ phase is studied by means of electron diffraction and high-resolution electron microscopy. A comparison is given between the results gained from this modification and the reported ones.

\section{Experiments}

The bulk crystal was grown with the vertical gradient freeze (VGF) method from the melt and synthesized directly in a quartz ampoule [BEILHARZ]. The feeding of highly pure Se followed after the alloying of metals with defined specifications in the ampoule. In order to optimize the heat exchanging process between furnace and material the ampoule were filled with Ar under 700 mbar at ambient temperature. Additionally, this Ar atmosphere prevented the formation of bubbles in the melt. The material synthesizing process was executed by the horizontal placement of the ampoule in a furnace. In the ampoule the synthesized material was present as ingots with a size of several $\mathrm{mm}^{3}$ to $\mathrm{cm}^{3}$. The ampoule with melted material was placed in the temperature field of a vertical furnace arrangement and moved over the solidification temperature until the total crystallization of material in the ampoule. The material was melted for 12 hours and homogenized at $50 \mathrm{~K}$ above the melting for additional 12 hours. The controllable solidifying and cooling process required 30 days. The growth speed was approximately $3 \mathrm{~cm}$ per day.

The crystal has a layered structure, which is even visible in a normal light microscope. The adhesion between the layers is weak, the hardness of the compound is low. 
Therefore, the conventional specimen preparation for TEM investigations is difficult. Specimens were prepared in two orientations, one parallel (plan-view) and one perpendicular to the layers (cross-section). For the first one, a tiny piece of the bulk crystal was introduced in a small glass tube filled with acetone, which was then brought in an ultrasonic bath. Under the influence of ultrasonic with a frequency of about $35 \mathrm{kHz}$ the layers felt apart so that some thin single layers could be captured on an amorphous carbonic mesh (Fig. 1).

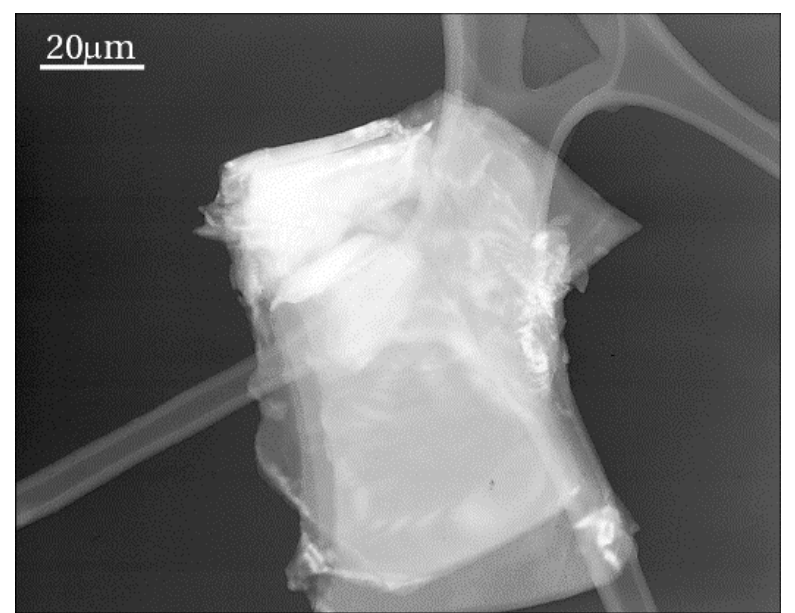

Fig. 1: TEM dark-field image of a single layer of CuIn5Se8 on an amorphous carbonic mesh

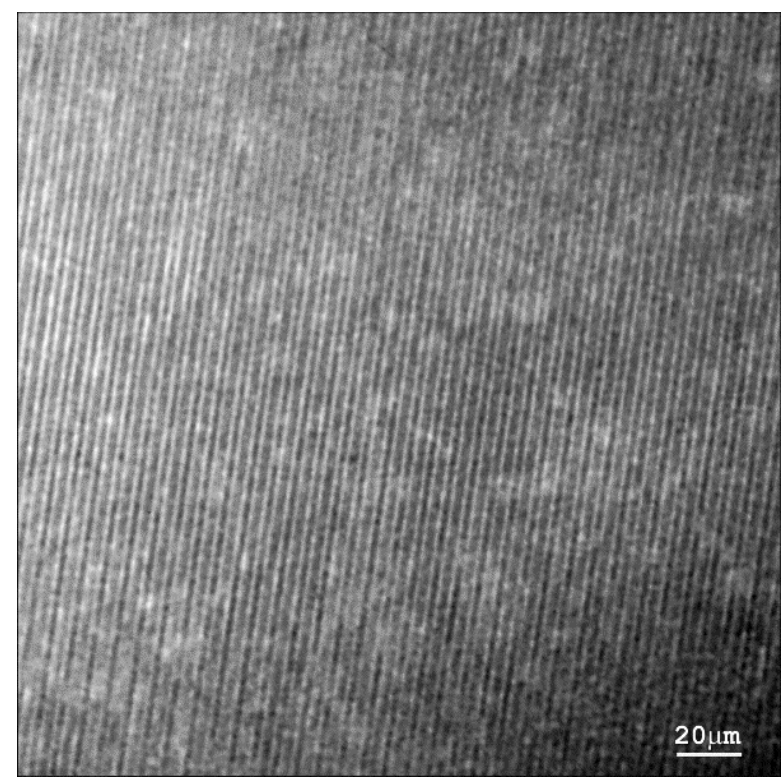

Fig. 2: TEM - Overview of the layered structure.

The second specimen was prepared perpendicularly to the layers. A small piece of the bulk crystal was embedded in resin and mechanically pre-thinned stepwise with diamond foils of different roughness down to the thickness of about 15-20 $\mu \mathrm{m}$, followed by ion-etching down to electron transparency using the GATAN 600 and BALTEC RES 100 ion milling machines (Fig. 2). TEM experiments were performed on various PHILIPS microscopes (EM400, CM12 at $120 \mathrm{kV}$ and CM200 FEG at $200 \mathrm{kV}$ ).

\section{Results and discussion}

The structure of the tetragonal $\alpha$-phase of $\mathrm{CuIn}_{5} \mathrm{Se}_{8}$ has already been studied and was found to be a tetragonal thiogallate structure with $\mathrm{c} \approx 2 \mathrm{a}$, which is a superstructure based on the zincblende structure. The ordering of the cations and of the vacancies in the tetrahedral interstitials of the cubic closed packed selenium sublattice causes the doubling of the unit cell along the c-axis [MANOLIKAS].
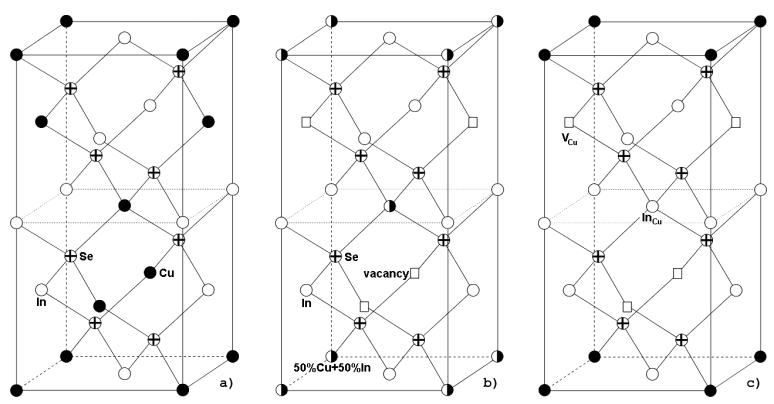

Fig. 3: Structure models of a) $\mathrm{CuInSe}_{2}$, b) $\mathrm{CuIn}_{5} \mathrm{Se}_{8}$ [MANOLIKAS] and c) $\mathrm{CuIn}_{5} \mathrm{Se}_{8}$ [WEI].

There are two known structure models of this $\alpha$ phase of $\mathrm{CuIn}_{5} \mathrm{Se}_{8}$, one of Manolikas et al. and the other one of Wei et al. shown in Fig. 3 ([WEI] [ZHANG]). In the structure model of Manolikas et al. the $(0,0,0)$ and the $(1 / 2,1 / 2,1 / 2)$ sites are statistically occupied by the cations (probability $50 \%$ of $\mathrm{Cu}$ and $50 \%$ of In atoms). The vacancies are located on the $(0,1 / 2,1 / 4)$ and $(1 / 2,0,3 / 4)$ sites (Fig. $3 b)$.

The structure belongs to the space group $I \overline{4}$ and has to obey the extinction condition $\mathrm{h}+\mathrm{k}+\mathrm{l}=2 \mathrm{n}$. The other structure model reported by Wei et al. was constructed, based on the introduction of the so-called defect pairs $2 \mathrm{~V}_{\mathrm{Cu}}{ }^{+}$ $+\mathrm{In}_{\mathrm{Cu}}{ }^{2-}$, where $\mathrm{V}_{\mathrm{Cu}}{ }^{+}$the vacancies of $\mathrm{Cu}$ atoms are on the $(0,1 / 2,1 / 4)$ and the $(1 / 2,0,3 / 4)$ sites and the antisite defects $\mathrm{In}_{\mathrm{Cu}}{ }^{2-}$ on the $(1 / 2,1 / 2,1 / 2)$ sites. It has the space group $P \overline{4}$ (Fig. 3c). A detailed description of the structure of these models was given elsewhere [THAM].

Fig. 4 shows electron diffraction patterns taken from plan-view and cross-section samples. They can be identified as diffraction patterns from the $\langle 110\rangle_{\mathrm{c}},\langle 112\rangle_{\mathrm{c}},\langle 221\rangle_{\mathrm{t}}$ and $<111\rangle_{\mathrm{t}}$ zone, where ${ }_{\mathrm{c}}$ and ${ }_{\mathrm{t}}$ are cubic and tetragonal indices, which are introduced in order to compare the corresponding diffraction patterns from the cubic (zincblende) and the tetragonal (thiogallate) unit cells. The relationship between the both indices is given as follows: $[\mathrm{uvw}]_{\mathrm{c}}=\left[\mathrm{uvw}_{/ 2}\right]_{\mathrm{t}}$ and $(\mathrm{hkl})_{\mathrm{c}}=(\mathrm{hk} 2 \mathrm{l})_{\mathrm{t}}$. The periodicity of 14 reflections in rows are visible in the patterns of the $\langle 110\rangle_{\mathrm{c}}$ and $\langle 112\rangle_{\mathrm{c}}$ zones (Fig. $4 a, 4 b)$, which were recorded with the electron beam parallel to the layers. They contain an alternative ordering of 7 stronger and 7 weaker reflections. In the perpendicular direction the patterns in the $\langle 221\rangle_{t}$ and $\langle 111\rangle_{\text {t }}$ zones show similar positions of reflections caused by the anion sublattice of the stoichiometric $\mathrm{CuInSe}_{2}$. It means that the same ordering of anions in the close-packed planes is given. The ordered presence of additional reflections gives evidence of 
a different distribution of cations and vacancies in the planes as in $\mathrm{CuInSe}_{2}$ [THAM].

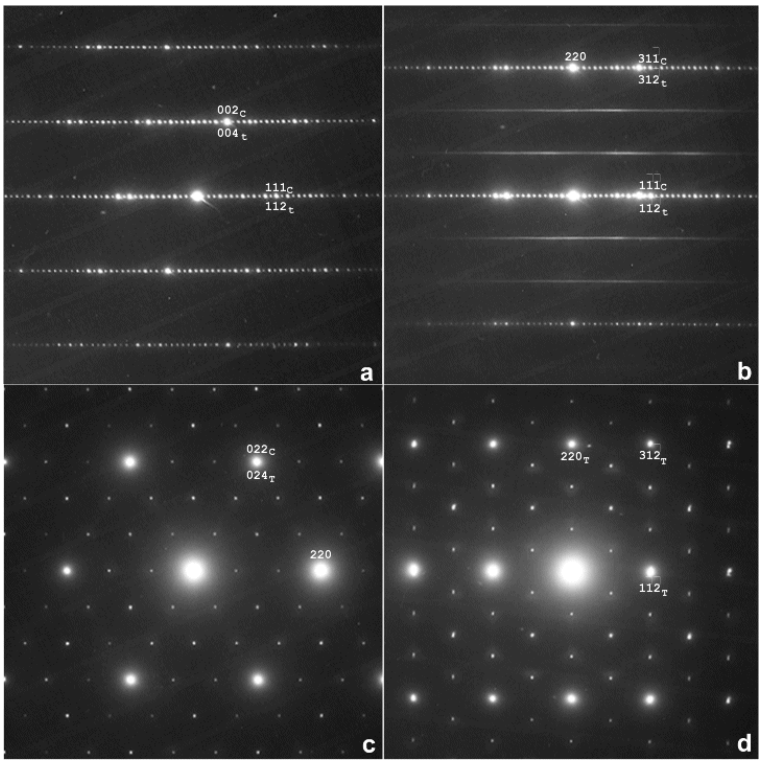

Fig. 4: Experimental electron diffraction patterns of the $\mathrm{CuIn}_{5} \mathrm{Se}_{8}$ compound in a) $<110>_{\mathrm{c}}$, b) $<112>_{\mathrm{c}}$, c) $<221>_{\mathrm{t}}$ and d) $<111>_{\text {t. }}$.

A high-resolution image of the layered structure of $\mathrm{CuIn}_{5} \mathrm{Se}_{8}$ is shown in Fig. 5. The image reveals unambiguously the stacking sequence of a 7-layer structure, which can be easily derived from shifts in the stacking of close-packed planes after every seventh plane. The presence of the other 7 weaker reflections in $\langle 110\rangle_{\mathrm{c}}$ and $\langle 112\rangle_{\mathrm{c}}$ patterns could be explained as an effect of the multiple scattering and pretends a 14-layer stacking sequence. The observed 7-layer sequence is given below as a mixed cubic-hexagonal stacking sequence and describes a polytype structure of $\beta$-phase $\mathrm{CuIn}_{5} \mathrm{Se}_{8}[\mathrm{TSENG}]$ :

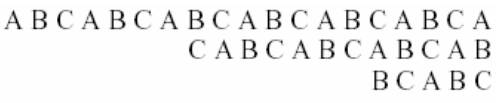

$\triangle B C A B \underline{C A} \cdot C A B C A \underline{B C} B$ C A B C

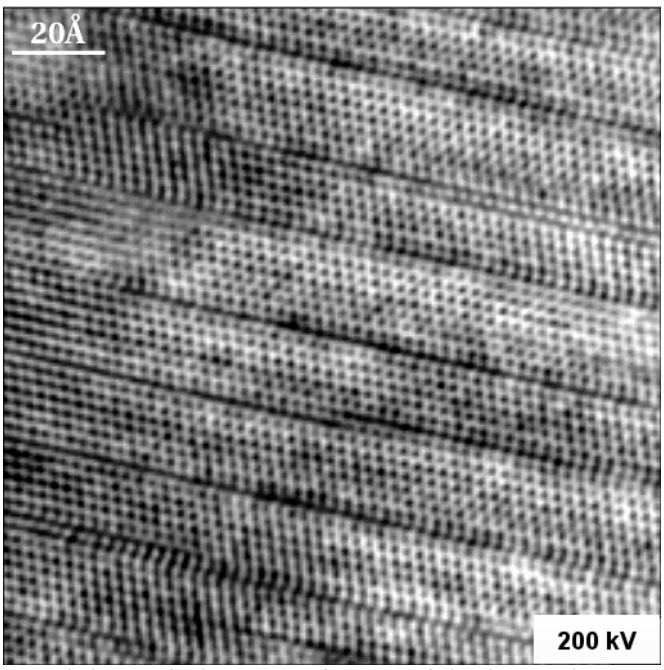

Fig. 5: High-resolution TEM image of the layered $\mathrm{CuIn}_{5} \mathrm{Se}_{8}$.

Fig. $4 \mathrm{~b}$ shows the appearance of two continuously

streaked rows of the reflections between the rows of sharp spots in equal $1 / 3$ distances, caused by the disordering of cations in the close-packed planes (in contrast to the ordering of cations in the tetragonal $\alpha$-phase $\mathrm{CuIn}_{5} \mathrm{Se}_{8}$ ). Similar results were observed and reported by Frangis et al. on another polytype of the $\beta$-phase $\mathrm{CuIn}_{5} \mathrm{Se}_{8}$ with a 5 -layer stacking sequence [FRANGIS]. In in-situ beam heating experiments with the illumination parallel to the $\langle 112\rangle_{c}$ direction the continuous streaks disappear slowly. A further heating makes to appear the rows again hinting to a phase transformation. This is contrary to the remarks of Frangis et al. that the streaks will be transformed in rows of discrete spots.

\section{Conclusion}

The studied material is a polytype of the $\beta$-phase $\mathrm{CuIn}_{5} \mathrm{Se}_{8}$ with 7-layer stacking sequence of the close-packed anion layers, which can be described as a mixed sequence of cubic and hexagonal stacking. In the layers the cations are disordered.

This work describes structural peculiarities of $\mathrm{CuIn}_{5} \mathrm{Se}_{8}$, characterized by means of electron diffraction and high-resolution electron microscopy. The experimental data are correlated with the structure models described in the literature.

\section{References}

BeIlHarz, C.: Ph. D. Thesis, University of Freiburg (1998).

Bloss, W. H., Pfisterer, F., Schubert, M., And Walter, T.: Progress in Photovoltaics: Research and Applications, 3, 3-24 (1995).

Frangis, N., Van Tedeloo, G., Manolikas, C., VAn Landuyt, J., AND Amelinckx, S.: Phys. Stat. Sol. (a), 96, 53-65 (1986). 